\title{
Research on ZigBee Technology in Mountainous Agriculture
}

\author{
Bin Chen ${ }^{1,2}$, Yijun $\mathrm{He}^{3}$, Zhenyi Zhao ${ }^{1,2}$ and Xiaojun Cui ${ }^{1,2}$ \\ ${ }^{1}$ Wenzhou Vocational College of Science and Technology, Wenzhou ZheJiang \\ 325006, China; \\ ${ }^{2}$ Wenzhou Academy of Agricultural Sciences, Wenzhou ZheJiang 325006, China; \\ ${ }^{3}$ Department of Information Science and Technology, Criminal Justice College, \\ East China University of Political Science and Law, Shanghai 201610, China
}

\begin{abstract}
In recent years, China vigorously has developed precision agriculture, but encountered a number of problems. For example, there are a lot of agricultural bases in the mountains in China, how to carry out the development of precision agriculture in the mountains is a difficult problem. In this paper, we design a fine agricultural wireless monitoring system based on ZigBee wireless sensor network which is suitable for mountain agriculture by using ZigBee technology. The system realizes the real-time collection of the environmental parameters of the unattended agricultural base. The system adopts multilevel tree cluster network architecture. Users can access the system at any time and anywhere through the smart phone or PC computer.
\end{abstract}

Keywords: mountainous agriculture; ZigBee; precision agriculture; multilevel tree cluster

\section{Introduction}

A great part of agricultural base in our country is in the mountains. And precision agriculture need real-time monitor on parameter such as temperature, humidity, illumination. For agricultural bases in remote mountainous areas, if traditional manual monitoring means to carry out, the difficulty is very big, is also not desirable.

In this paper, researcher designed a fine agriculture wireless monitoring system. The system is based on ZigBee wireless sensor network [1]. It collected the data of mountain agricultural base, such as temperature, humidity, illumination. The system supported long-term work, intelligent operation, unattended, etc.

\section{Overall Structure of the System}

The overall architecture of this system is shown in figure 1. ZigBee node can be divided into three roles, such as Coordinator, Router, End Device. Researcher need to build a multi-level tree cluster structure of ZigBee wireless sensor network in each agricultural base which includes a coordinator node, multiple routers, numerous terminal nodes. Each router node and its child nodes form a cluster. If the child node is a router, the child nodes form a cluster. So, form a multi-level tree cluster network. Temperature, humidity, light intensity and other sensors are configured on the acquisition terminal node to realize real-time monitoring of various parameters such as temperature, humidity, light and so on. It is also equipped with solar modules for continuous power supply, so that the node can continue to work. The embedded gateway is responsible for the data interaction between the wireless sensor network and server. It collected data and sent to the cloud server according to the instruction. Researcher developed web application management system based on three-layer architecture. Users can use PC and smart phone to login the 
system through the internet and monitor of the specified agricultural base at any time any place.

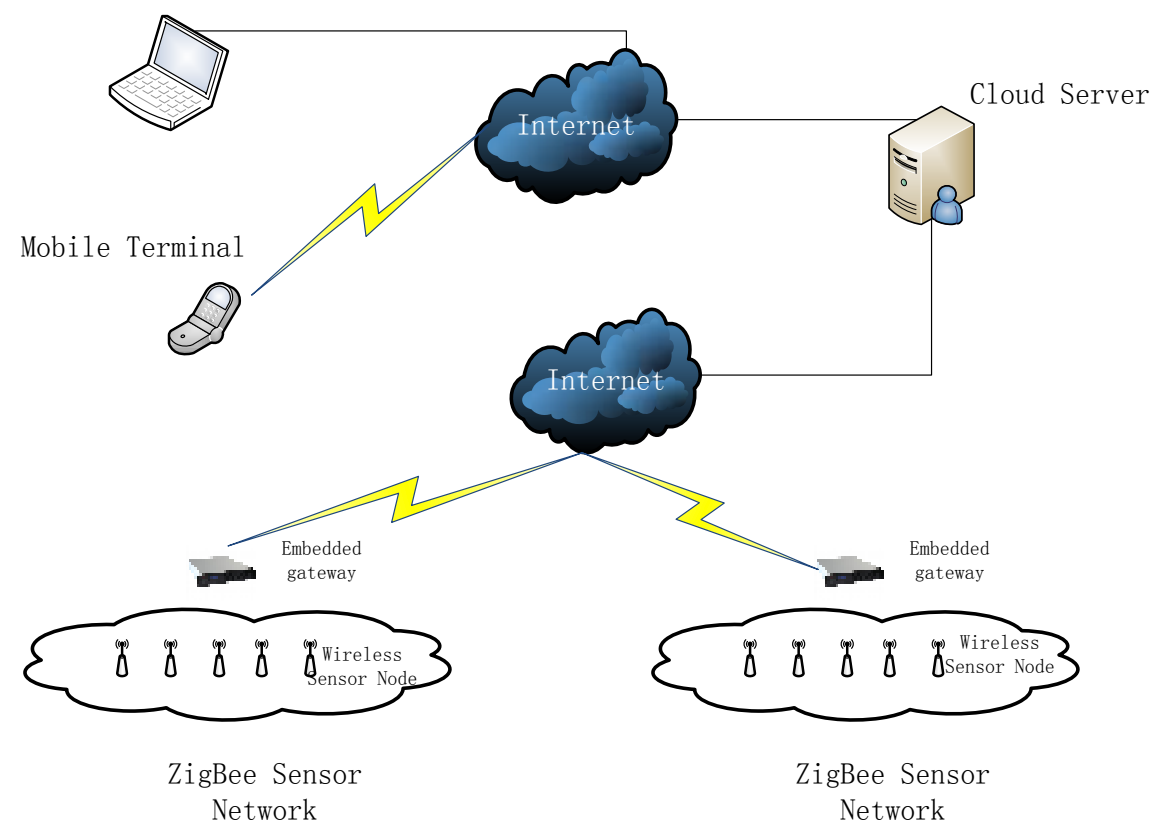

Figure 1. System Architecture Diagram

\section{The System Hardware Design}

\subsection{Selection of the Chip}

Sensor node chip using CC2530 company's TI chip. The chip is compatible with the IEEE802.15.4 chip system, which supports the proprietary 802.15.4 market, ZigBee, ZigBeePRO, and ZigBeeRF4CE standards. It provides the $101 \mathrm{~dB}$ link quality with strong anti-interference and excellent receiver sensitivity. It also has a variety of power supply mode, and a set of a wide range of peripherals set, including two USAR, 12-bit ADC and 21 GPIO. It has excellent RF performance, equipped with standard enhanced $8051 \mathrm{MCU}$ core, also supports the low power wireless communication.

CC2530 can be applied to many occasions, which includes smart home appliance control, remote control, consumer electronics, measurement and intelligent energy, building automation, medical and other fields. According to the different capacity of built-in memory chips, $\mathrm{CC} 2530$ has three different versions. Compared with the first generation of $\mathrm{CC} 2430, \mathrm{CC} 2530$ provides a powerful address recognition and packet processing engine. It can be a good to match RF front-end, and encapsulation smaller, and support for ZigBeePRO and ZigBeeRF4CE. The inside is divided into 3 categories: CPU and related memory module, peripheral, clock and power management module, wireless module.

\subsection{Wireless Sensor Network Node Hardware Design}

In this system, terminal node and the router use the same hardware structure and been given different roles. Node runs the function of the corresponding program according to its own role. Wireless sensor network node includes wireless communication module, sensor module, power panel module components, as shown in figure 2 .

(1) Wireless communication module. The communication distance between sensor nodes within 10 meters usually can meet the needs of the system in Greenhouses [2]. And in the wild mountain environment is a clear need for further effective communication 
range to meet the actual needs. In order to adapt to the mountainous environment and increase the coverage of sensor, researcher chooses a relatively large power inverted $\mathrm{F}$ antenna.

(2) Sensing module, including temperature sensing module, humidity, light sensing module, etc. [3] Different agricultural bases, because of different agricultural products, need to monitor different environmental parameters. Researcher can join kinds of sensors by extending the bus [4]. Researcher chooses SHT11 as temperature and humidity sensor module. SHT11 is patch type temperature and humidity sensor. It possesses a full range of calibration, two-line digital output; Humidity measurement range: $0 \sim 100 \%$ RH; Temperature measurement range: $-40 \sim+123.8{ }^{\circ} \mathrm{C}$; Humidity measurement accuracy: $\pm 3 \% \mathrm{RH}$; Temperature measuring accuracy: $\pm 0.4{ }^{\circ} \mathrm{C}$; Response time: 8 (tau63\% s); Low power $80 \mu \mathrm{W}$; It can be completely submerged.

(3) Power supply board, which realizes the connection between the node module and the sensing module, and realizes the system power supply, can be powered by two sections of batteries. It can be directly supported by the dc power supply, can also be connected to solar energy power supply module. The solar energy power supply can solve the problem of long time unattended in the field, and is also energy-saving and environmental protection. This system is aimed at the mountain environment, so equipped with relatively large solar panels [5]. Especially the coordinator and router nodes are also equipped with bigger solar panels, which are responsible for more tasks.

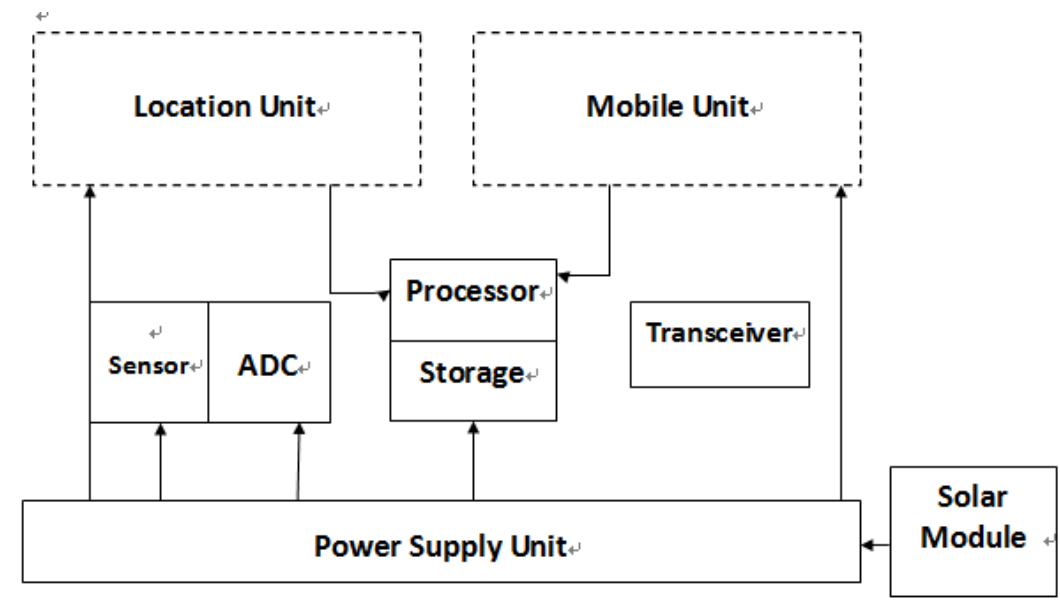

Figure 2. Hardware Structure Diagram of Wireless Sensor Network Node

\section{The System Software Design}

\subsection{Sensor Node Protocol Stack}

This protocol stack of sensor node in the system is a rotary type operating system queries based on protocol stack. It is composed of the operating system abstraction layer (OSAL) and the various layers of the ZigBee protocol which including the hardware abstraction layer (HAL), MAC layer, network layer, ZDO layer, application framework layer (AF). The Z-Stack protocol stack and OSAL are independent of each other. The ZStack protocol stack is relied on OSAL to run. OSAL is a multi tasking, non preemptive, polling resource management mechanism of the operating system.

Each layer of ZigBee can be used as a task in the Z-Stack protocol stack to run independently. OSAL uses a linked list structure to manage the corresponding task of each layer of the protocol stack. The linked list of task is shown in figure 3. Each item in the list is a structure that is used to record information about the related tasks in the list. The establishment of the list is in accordance with the order of task priority from high to 
low. A high priority task will be inserted into the front of a low priority task. If two tasks have the same priority, they are added to the list in chronological order. The task list is set up at system startup time. And it is destroyed when the system is closed or hardware reset.

\begin{tabular}{|c|}
\hline Task(n)-------SampleApp_loop \\
\hline ........ \\
\hline Task2--------Hal_ProcessEvent \\
\hline Task1--------nwk_event_loop \\
\hline Task0--------macEventLoop \\
\hline
\end{tabular}

Figure 3. Chart of Task List

1. The system initialization

Startup code needs to complete the initialization of hardware platform and software architecture. It should prepare for the operation of the operating system, including test chips working voltage, the initialization of the system clock, the memory stack, each hardware module, FLASH storage, MAC address, chip initialization nonvolatile variables and the MAC layer protocol and so on.

After prepare for execution of the operating system to work, Startup code begin to execute operating system entry procedures and returns the control to the operating system completely.

2. Task event implementation

OSAL shields the communication primitives of the physical layer, MAC layer and network layer. The development of Z-Stack protocol stack can be simplified to the program development based on OSAL. Message response and task scheduling are implemented by OSAL. There are two ways to trigger the task event, one is calling the system message passing mechanism, and the other is the soft timer osal_start_timerEx (). When there is an event to handle, OSAL call the appropriate event handler.

\subsection{Software Design of the Coordinator Node}

According to the system design scheme, the coordinator should not only responsible for the establishment of wireless sensor network, but also responsible for network maintenance. It transmits network beacon, manages network nodes and storages data, provides the routing information of associated node between the table [6].

Coordinator node because of the heavy task, so the relative to other nodes, it has a more powerful hardware resources and stable and reliable power supply, the use of direct current power supply and solar powered dual channel. After building the network, the coordinator node chooses a network identifier for this network and its network address (short) is set to 0 . Then it starts to send its beacon to neighboring nodes and accepts connection from other nodes. So the first level of the tree topology is set up. The relationship between coordinator and these nodes is parent-child relationships, and the network nodes are allocated a 16-bit network address. The software design of coordinator node is shown in figure 4. 


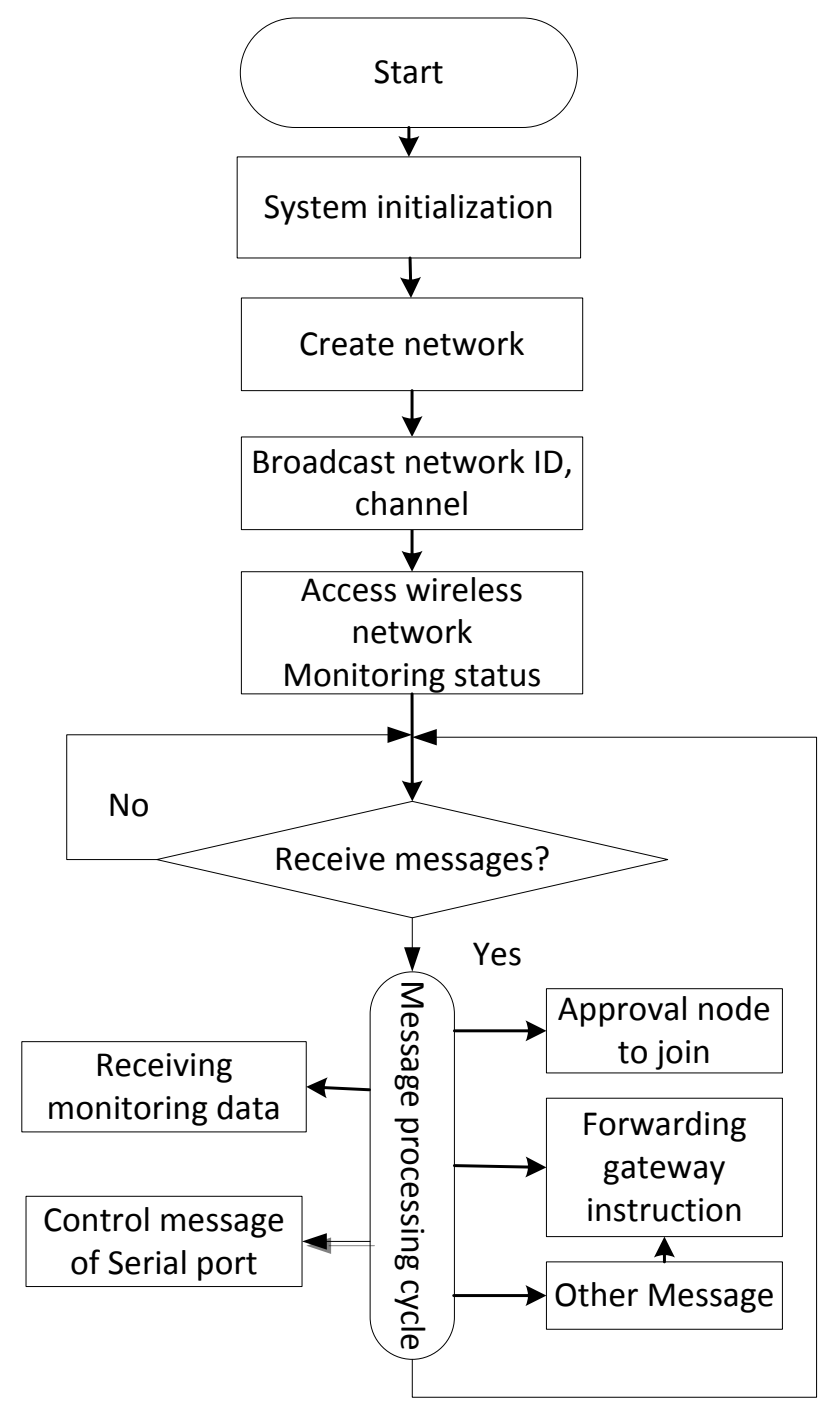

Figure 4. Flow Chart of the Coordinator Node

\subsection{Software Design of Terminal Nodes}

According to the system design scheme, the system uses terminal node as data collection nodes. After system boot, the coordinator will allocate a unique 16 network address for terminal node when it accesses networks. It sends the collected data to the own routing nodes (the parent node). The routing nodes forward the data to the coordinator node. At last, the coordinator nodes send the data to the data server in the internet web.

In the field, the power supply of sensor network nodes is a difficult problem. Even using solar power, researcher also should consider the issue of energy consumption. If the weather is not good for a few days, it is easy for a node to shut down because there is no energy. So we used the wake up sleep mode. When the terminal node is required to collect environmental data, the system will wake up the node. After the data is collected, the node is in a state of sleep, waiting for the next wake-up time. Researcher improves the effectiveness of using electrical energy, and extend the working hours. The software design flow chart of the terminal node is shown in Figure 5. 


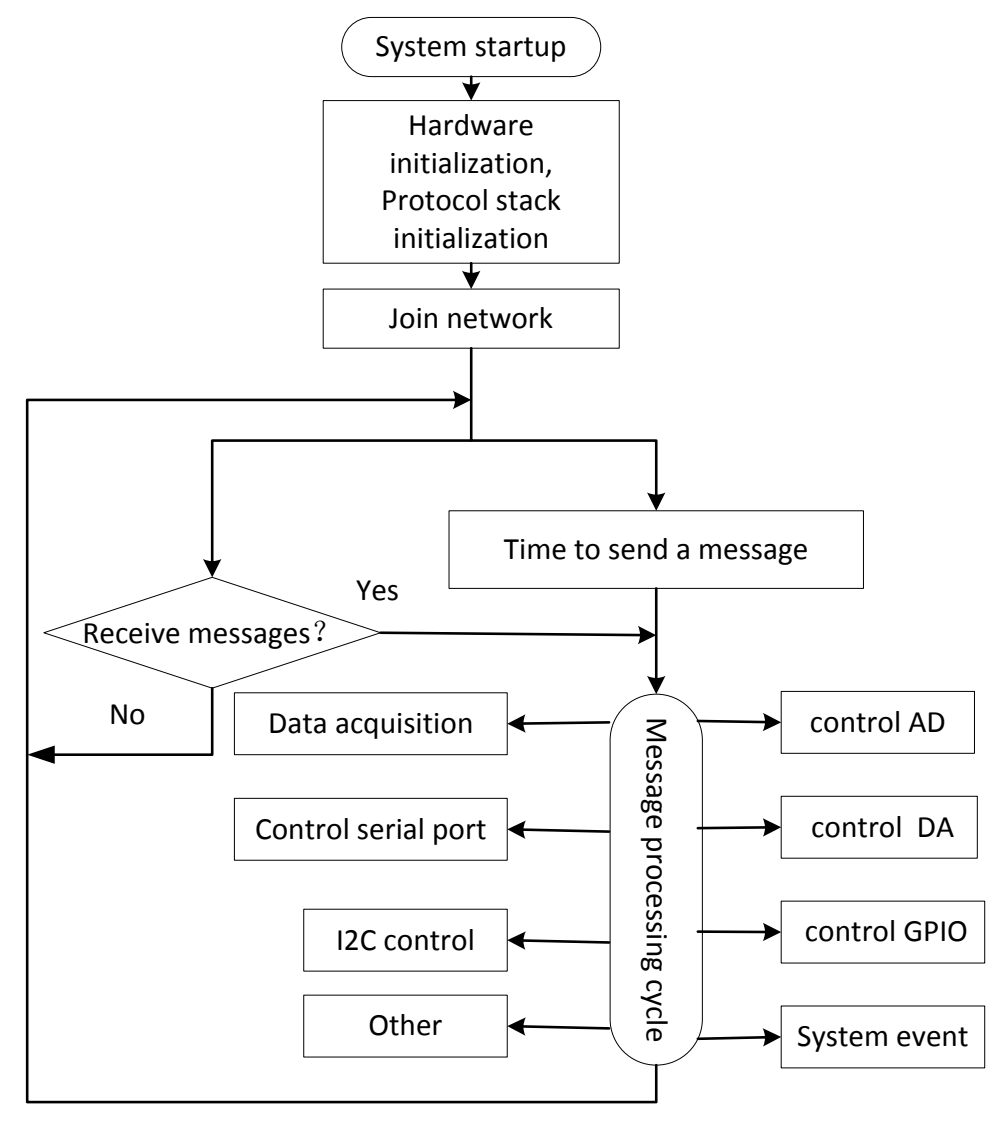

Figure 5. Flow Chart of Terminal Node

The data packet sent by each terminal node can be represented by a structure, including the device type, the network address, the address of the parent node and the data collected by the node, as shown in Table 1.

Table 1. Data Packet Structure

\begin{tabular}{llllll}
\hline Head & $\begin{array}{l}\text { Device } \\
\text { Type }\end{array}$ & $\begin{array}{l}\text { Node } \\
\text { Address }\end{array}$ & $\begin{array}{l}\text { Parent } \\
\text { Node } \\
\text { Address }\end{array}$ & $\begin{array}{l}\text { Acquisition } \\
\text { Data }\end{array}$ & Tail \\
& & & & \\
\hline
\end{tabular}

\subsection{Routing Node Program Execution Flow}

The function of ZigBee router node is to allow other devices to join the network, multi hop routing. When the router node starts, it will connect with the coordinator. Coordinator will assign an address block (including a number of 16-bit network address [7]) for it. The router is configured and sent its own beacon based on the information it receives from the coordinator beacon, allowing other devices to connect with their own. These devices will become its child nodes. These sub nodes may be terminal node or the router node. The router node can also have its own set of child nodes, in this way to expand indefinitely formed multistage cluster tree structure network [8]. The software design flow chart of routing node is shown in Figure 6. 


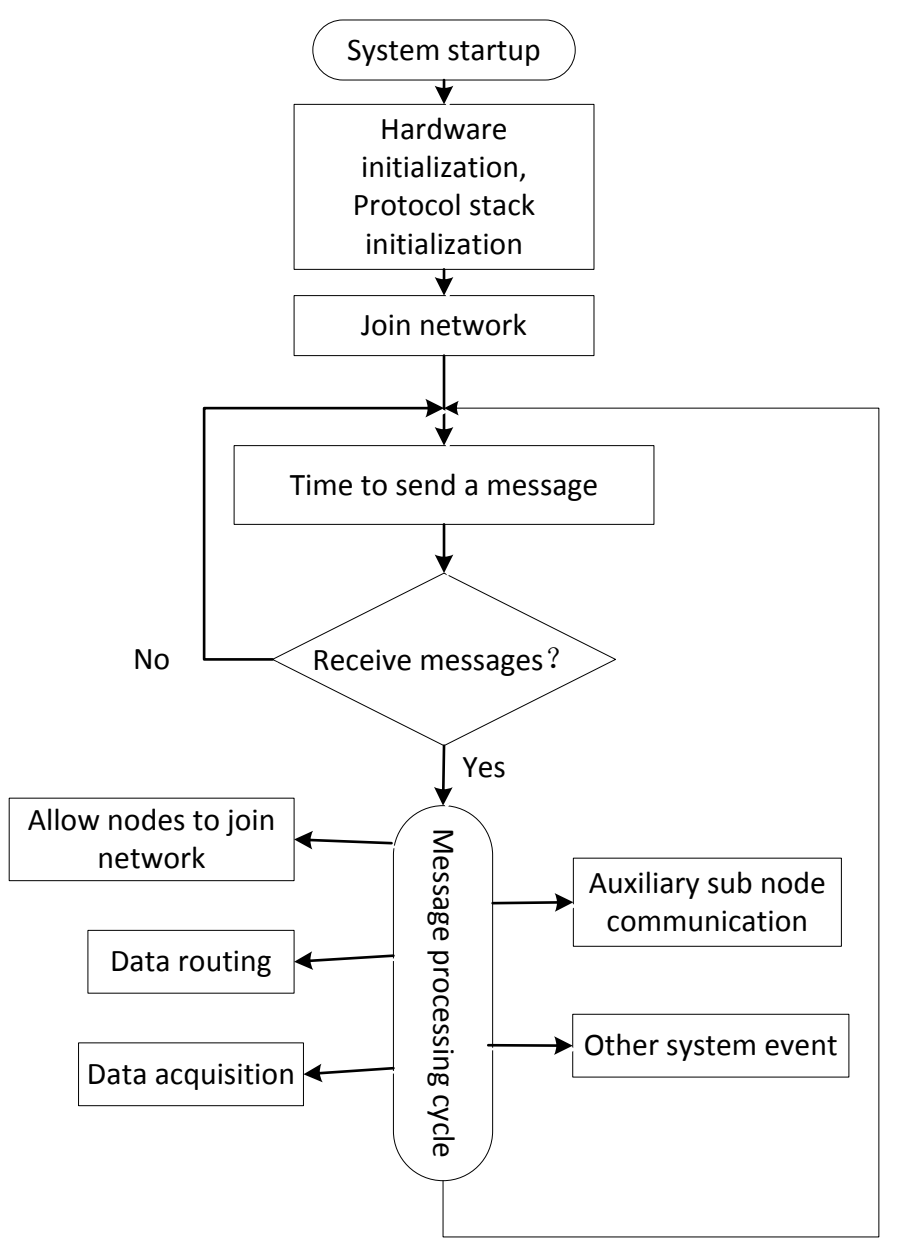

Figure 6. Routing Node Program Execution Flow Chart

\subsection{Design of Upper Layer Application Management Software}

The upper layer application management software adopts the three-layer B/S model, which is convenient for maintenance and update. Application management software provides a variety of functions, such as real-time graph, node resource list, statistical report and so on, to meet the needs of users [9]. Users can at any time and any place to use a smart phone or PC computer, login system, real-time remote monitoring of agricultural base [10]. If the abnormal situation, the system will be short message, telephone and other means to the user real-time alarm. The running interface of the upper application management software is shown in Figure 7. 


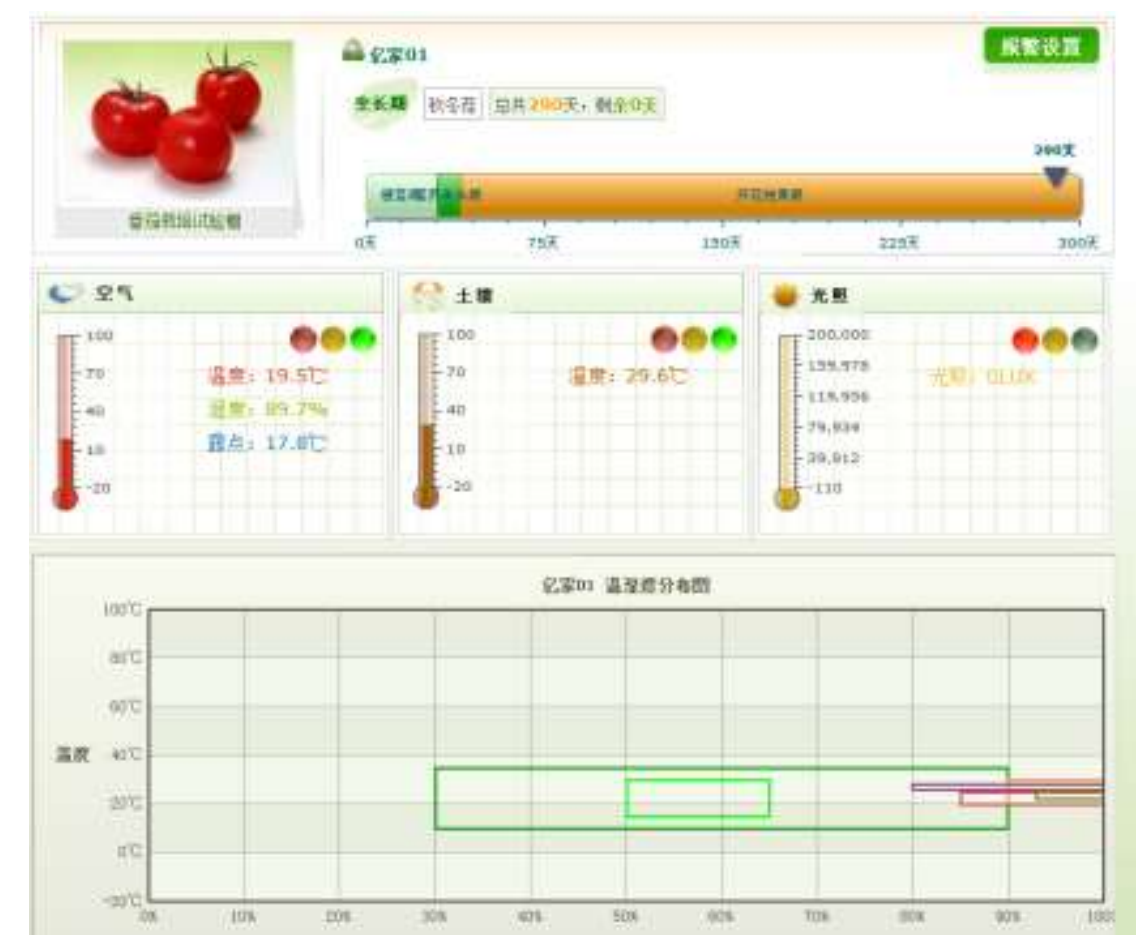

Figure 7. Software Running Interface Diagram

\section{Concluding Remarks}

In this paper, the design of for in a mountainous area agriculture base fine agricultural wireless monitoring system, in the design of the network using multistage cluster tree type structure, sensor nodes as a source of energy with solar charging technology, an effective solution to the large areas in remote mountainous areas unattended agricultural base environmental parameters real-time acquisition and monitoring problems. Users can access the system at any time and any place by using smart phones or PC computers to realize the remote real-time monitoring of agricultural base. System for monitoring the abnormal situation, such as the temperature is too low, the humidity is too high and other situation, will automatically text messages, phone calls to the user in real-time alarm. The research and development of the system has a certain positive significance to the development and promotion of fine agriculture.

\section{Acknowledgements}

This work was financially supported by 2016 Annual Educational Technology Research Program of Zhejiang Province (NO.JA066), National education information technology research project (NO. 146231592), the Public Welfare Research Project of Zhejiang Province (No. 2014C32031), the Public Welfare Science and Technology Project of Wenzhou City (No. N20140047).

\section{References}

[1] S. Farahani, "ZigBee Wireless Networks and Transceiver", USA, Newnes, (2008).

[2] W. Yinling and S. Tao, "The Design of Greenhouse Environmental Monitoring of Wireless Sensor Network Nod", Journal of Agricultural Mechanization Research, vol. 33, no. 4, (2011), pp. 113-117.

[3] W. Yinfang and M. Shiwei, "Research and Design of Wireless Meter Reading System Based on ZigBee”, Measurement and control technology, vol. 32, no. 1, (2013), pp. 14-18.

[4] W. Wencheng and C. Faliang, "Wireless Measurement and Control System of Temperature and Humidity in Greenhouse", Instrument technique and sensor, vol. 35, no. 3, (2011), pp. 98-103. 
[5] Y. Zhiqiang, "Design of wireless monitoring system for greenhouse based on ZigBee Technology", Jiangsu agricultural sciences, vol. 40, no. 11, (2012), pp. 396-397.

[6] Z. Baozhou, L. Fuqiang and W. Lili, "Design of storage environment monitoring system based on ZigBee and technology GPRS", Journal of agricultural science and technology of Jiangxi, vol. 25, no. 5, (2013), pp. 107-110.

[7] L. Yunfei, "Research on address shortage in a tree based ZigBee network", Int'l Conf on Information Security and Intelligence Control (ISIC), Beijing, China, (2012).

[8] G. Yipeng, L. Ziming and W. Zelin, "Design of multi point strain monitoring system based on technology ZigBee", informationization of transportation, vol. 23, no. 2, (2013), pp. 157-179.

[9] C. Zhiwei, S. Wejun and Y. Chongchong, "Design of agricultural greenhouse monitoring system based on WSNs", Sensor and micro system, vol. 30, no. 7, (2011), pp. 82-87.

[10] Z. Xiaobin, Z. Kefeng and Z. Jiancheng, "Discussion on Application of wireless sensor network in Greenhouse in Zhejiang”, Journal of agriculture in Zhejiang, vol. 20, no. 3, (2011), pp. 25-28.

\section{Authors}

Bin Chen (1981.11-) Lecturer, Master, Research Orientation: Wireless sensor network.

Yijun He (1981.12-) Lecturer, Doctor, Research Orientation: Information Safety.

Zhenyi Zhao (1985.01-) Lecturer, Master, Research Orientation: Wireless sensor network, Electronic Commerce.

Xiaojun Cui (1972.02-) Professor, Doctor, Research Orientation: Wireless sensor network. 
International Journal of Future Generation Communication and Networking Vol. 10, No. 1 (2017) 